\title{
Utilizing the Natural Fiber for Textile and Engineering
}

\section{Ashish Chauhan*}

Ministry of Chemical and Fertilizer, National Institute of Pharmaceutical Education and Research (NIPER), India

Nowadays, the whole world has focused its attention towards renewable and sustainable resources because of environment and health concerns. The development of new polymer from renewable raw materials in comparison to artificial fibers has increased during last few years. Natural fibers like pine needle, flax, jute and ramie are the most suitable reinforcement materials because of their relatively high strength and stiffness in industries such as automobile, packaging and construction materials due to economic and ecological aspects. Low cost, easily availability and required properties (viz. low density, mechanical properties) makes them attractive in place of glass, carbon and other synthetic fibers. The constituents of natural fibers are cellulose, hemicelluloses, lignin and pectins with a small quantity of the extractives. These natural fibers such as cellulose and silk are of tremendous use in textile industry. The properties of bio-fibers mainly depend upon their origin, age, climatic conditions and extraction techniques. The presence of hydroxyl groups (polar group) in various constituents of lingo-cellulosic fiber reduces the utilities in many applications. In order to improve its adhesion, various techniques like graft copolymerization, chemical treatment (viz. mercerization, acetylation, benzoylation etc.) and treatment with various coupling agent can be used. Graft copolymerization is an efficient technique to impart desirable properties to backbone polymers. Various workers have carried out the graft copolymerization onto different cellulosic backbone using vinyl monomers through various chemical and radiation techniques. Grafting of acrylate monomers individually and in binary mixtures onto cotton yarn by emulsion technique initiated by ceric ions has been widely studied. Graft copolymerization of methyl acrylate onto cellulose and thiolated holocellulose reported decrease in incorporation of graft in the latter. Graft copolymerization can be initiated by using chemical, ionic and radical initiator systems. Among these initiation systems the chemical initiation by grafting involving oxidizing agents such as potassium permanganate, potassium bromate, ceric ammonium nitrate, ozone hydroxyl radicals etc are promising from economic point of view. Different workers have studied the kinetics of graft copolymerization of vinyl monomers onto the starch and synthetic copolymers but few attempts seems to have been made to study kinetics of graft copolymerization of vinyl monomers onto natural fibers. Graft copolymerization of acrylic monomers onto cotton fabric using an activated cellulose thiocarbonate-azobis-isobutyronitrile redox system has been investigated and it has been found that a reaction medium of $\mathrm{pH} 2.0$ and temperature of $70^{\circ} \mathrm{C}$ constituted the optimum conditions for grafting. Methyl Meth
Acrylate (MMA) has been found to be superior than other monomers. Chemically modified cellulosic depicts higher grafting with MMA as compared to unmodified cellulose. Oxidized cellulose was grafted with 2-methyl-5-vinyl pyridine using a $\mathrm{H}_{2} \mathrm{O}_{2}$-thioureadioxide redox system. Considerable grafting took place on oxidized cellulose on excluding thio-ureadioxide from the system whereas it offsets grafting onto cotton cellulose. Grafting of MMA onto modified cellulose at $60^{\circ} \mathrm{C}$ using peroxydiphosphate as an initiator, they determined the rate of grafting by varying initiator, monomer, nature of the substrate and temperature. MMA was graft copolymerized onto cotton-cellulose using hexavalent chromium, $\mathrm{Cr}$ (VI) as an initiator. Graft copolymers were subjected to various chemical, mechanical and thermal evaluation and the results showed that there has been a significant improvement in properties. Graft copolymerization of styrene onto cellulose was investigated under variable reaction conditions using $\mathrm{H}_{2} \mathrm{O}_{2}$ as an initiator. Cellulose carbamates having less than $1.1 \%$ nitrogen showed lower graft yield than the unmodified cellulose. Styrene was graft copolymerized onto cellulose in the form of cotton linters, cotton cloth and rayon by ionizing radiation. The graft copolymers thus obtained were characterized by removing the cellulose back-bone on hydrolysis and determining the molecular weights of the residual poly (styrene). Graft copolymerization has been widely studied using various vinyl monomers by different techniques and has improved various properties of the fiber. With the advancement of science and technology there are numerous advanced techniques like SEM, AFM, TEM, XRD, FTIR, DSC, TGA, DTA that can evaluate various morphological, physicochemico-thermal change in fiber.

We have seen above how the various researchers have utilized the low weight and high strength of natural fibers and polymers as backbone and graft copolymerized by various physico-chemical and radiation techniques using numerous monomers and characterization techniques to procure a number of modified graft copolymers. These graft copolymers are modified materials that can have desired physico-chemico-thermo-mechanical properties, depending upon the monomers and technique used. But, still a lot remains unexplored. Natural resources are the gift of the God to the mankind. But, with time these renewable resources and natural fiber will soon deplete. So, there is a great need to sustain and procure them for the future. They should be used economically and wisely. We should seek more fruitful means to explore the maximum potential and utilize the natural fiber for the development of science and technology.

*Corresponding author: $\mathrm{Dr}$. Ashish Chauhan, Ministry of Chemical and Fertilizer, National Institute of Pharmaceutical Education and Research, India, Tel: 919464616773; E-mail: aashishchauhan26@gmail.com

Received August 22, 2012; Accepted August 23, 2012; Published August 30 2012

Citation: Chauhan A (2012) Utilizing the Natural Fiber for Textile and Engineering $\mathrm{J}$ Textile Sci Eng 2:e108. doi:10.4172/2165-8064.1000e108

Copyright: (c) 2012 Chauhan A. This is an open-access article distributed unde the terms of the Creative Commons Attribution License, which permits unrestricted use, distribution, and reproduction in any medium, provided the original author and source are credited. 